\title{
Concurrent Multiscale Simulation at Finite Temperature: Coarse- Grained Molecular Dynamics
}

R.E. Rudd

This article is to appear in the Handbook of Materials Modeling.

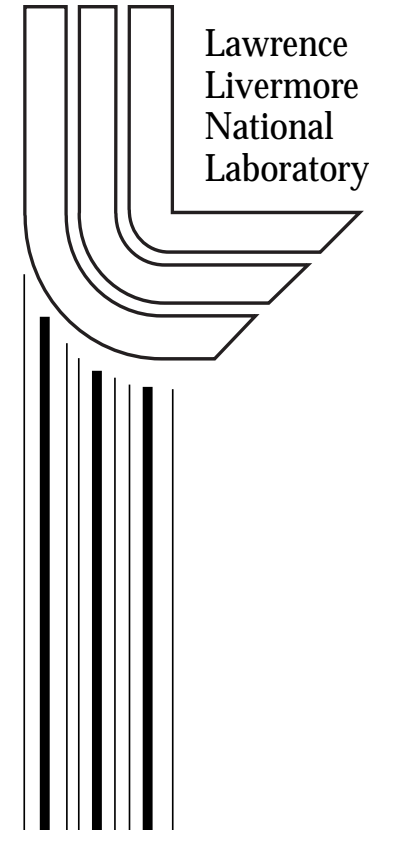

January 20, 2004 


\section{DISCLAIMER}

This document was prepared as an account of work sponsored by an agency of the United States Government. Neither the United States Government nor the University of California nor any of their employees, makes any warranty, express or implied, or assumes any legal liability or responsibility for the accuracy, completeness, or usefulness of any information, apparatus, product, or process disclosed, or represents that its use would not infringe privately owned rights. Reference herein to any specific commercial product, process, or service by trade name, trademark, manufacturer, or otherwise, does not necessarily constitute or imply its endorsement, recommendation, or favoring by the United States Government or the University of California. The views and opinions of authors expressed herein do not necessarily state or reflect those of the United States Government or the University of California, and shall not be used for advertising or product endorsement purposes.

This is a preprint of a paper intended for publication in a journal or proceedings. Since changes may be made before publication, this preprint is made available with the understanding that it will not be cited or reproduced without the permission of the author. 


\section{CONCURRENT MULTISCALE SIMULATION AT FINITE TEMPERATURE: COARSE-GRAINED MOLECULAR DYNAMICS}

\section{Embedded Nanomechanics and Computer Simulation}

With the advent of nanotechnology, predictive simulations of nanoscale systems have become in great demand. In some cases nanoscale systems can be simulated directly at the level of atoms. The atomistic techniques used range from models based on a quantum mechanical treatment of the electronic bonds to those based on more empirical descriptions of the interatomic forces. In many cases, however, even nanoscale systems are too big for a purely atomistic approach, typically because the nanoscale device is coupled to its surroundings, and it is necessary to simulate the entire system comprising billions of atoms. A well-known example is the growth of nanoscale epitaxial quantum dots in which the size, shape and location of the dot is affected by the elastic strain developed in a large volume of the substrate as well as the local atomic bonding. The natural solution is to model the surroundings with a more coarse-grained description, suitable for the intrinsically longer length scale. The challenge then is to develop the computational methodology suitable for this kind of concurrent multiscale modeling, one in which the simulated length scale can be changed smoothly and seamlessly from one region of the simulation to another while maintaining the fidelity of the relevant mechanics, dynamics and thermodynamics.

The realization that Nature has different relevant length scales goes back at least as far as Democritus. Some 24 centuries ago he put forward the idea that solid matter is comprised ultimately at small scales by a fundamental constituent that he termed an atom. Implicit in his philosophy was the idea that an understanding of the atom would lead to a more robust understanding of the macroscopic world around us. In the intervening period, of course, not only has the science of this atomistic picture been put on a sound footing through the inventions of chemistry, the discovery of the nucleus and the development of quantum mechanics and modern condensed matter physics, but a host of additional length scales with their own relevant physics has been uncovered. A great deal of scientific innovation has gone into the development of physical models to describe the phenomena observed at these individual length scales.

In the past decade a growing effort has been devoted to understanding how physics at different length scales works in concert to give rise to the observed behavior of solid materials. The use of models at multiple length scales, especially computer models optimized in this way, has become known as multiscale modeling. An example of multiscale modeling that we will consider in some detail is the modeling of the elastic deformation of solids at the atomistic and continuum levels. Clearly one kind of multiscale model would be to calculate the mass density and elastic constants within an atomistic model, and to use those data to parameterize a 
continuum model to describe large-scale elastic deformation. Such a parameter-passing, hierarchical approach has been used extensively to study a variety of systems (Moriarty et al., 2002). Its success relies on the occurrence of well-separated length scales. We shall refer to such an approach as sequential multiscale modeling.

In some systems, it is not clear how to separate the various length scales. An example would be turbulence, in which vortex structures are generated at many length scales and hierarchical models have to date only worked in very special cases (Townsend, 1976). Alternatively, the system of interest may be inhomogeneous and have regions in which small-scale physics dominates embedded in regions governed by large-scale physics. Examples would include fracture (Abraham et al., 2002, 1998), various nucleation phenomena (Mason et al., 2004), nanoscale moving mechanical components on computer chips (NEMS) (Rudd and Broughton, 1999), ion implantation and radiation damage events (Averback and Diaz de la Rubia, 1998), epitaxial quantum dot growth (Rudd et al., 2003) and so on. In either case hierarchical approach is not ideal, and concurrent multiscale modeling is preferred (Rudd and Broughton, 2000). Here we focus on the inhomogeneous systems, and in particular on systems like those mentioned above in which the most interesting behavior involves the mechanics of a nanoscale region, but the overall behavior also depends on how the nanoscale region is coupled to its large-scale surroundings. This embedded nanomechanics may be studied effectively with concurrent multiscale modeling, where regions dominated by different length scales are treated with different models, either explicitly through a hybrid approach or effectively through a derivative approach.

Here we focus on the methodology of Coarse-Grained Molecular Dynamics (CGMD) (Rudd and Broughton, 1998, 2000, 2004; Rudd, 2002), one example of a concurrent multiscale model. CGMD describes the dynamic behavior of solids concurrently at the atomistic level and at more coarse-grained levels. The coarse-grained description is similar to Finite Element Modeling (FEM) of continuum elasticity, with several important distinctions. CGMD is derived directly from the atomistic model without recourse to a continuum description. This approach is important because it allows a more seamless coupling of the atomistic and coarse-grained models. The other important distinction is that CGMD is designed for finite temperature, and the coarse-graining procedure makes use of the techniques of statistical mechanics to ensure that the model provides a robust description of the thermodynamics. Several other concurrent multiscale models for solids have been proposed and used (Kohlhoff et al., 1991; Tadmor et al., 1996; Broughton et al., 1999; Shilkrot et al., 2002; Curtarolo and Ceder, 2002; Curtin and Miller, 2003). The Quasicontinuum technique is of particular note in this context, because it too is derived entirely from the underlying atomistic model (Tadmor et al., 1996). CGMD was the first concurrent multiscale model designed for finite temperature simulations (Rudd and Broughton, 1998). Recently, another finite temperature concurrent multiscale model has been developed using renormalization group techniques, including time renormalization (Curtarolo and Ceder, 2002). This model is very interesting, 
although to date its formulation is based on bond decimation procedures that is limited to simple models with pair-wise nearest-neighbor interactions. The formulation of CGMD is more flexible, making it compatible with most classical interatomic potentials. It has been applied to realistic potentials in 3D whose range extends beyond nearest neighbors.

\section{Formulation of CGMD}

Coarse-grained molecular dynamics provides a model whose minimum length scale may vary from one location to another in the system. The CGMD formulation begins with a specification of a mesh that defines the length scales that will be represented in each region (see Figure 1). As in finite element modeling (Hughes, 2000), the mesh is unstructured, and it comes with a set of shape functions that define how fields are continuously interpolated on the mesh. For example, the displacement field is the most basic field in CGMD, and it is approximated as

$$
\mathbf{u}(\mathbf{x}) \approx \sum_{j} \mathbf{u}_{j} N_{j}(\mathbf{x})
$$

where $N_{j}(\mathbf{x})$ is the value of the $j^{t h}$ shape function evaluated at the point $\mathbf{x}$ in the undeformed (reference) configuration. It is often useful to let $N_{j}(\mathbf{x})$ have support at node $j$ so that the coefficient $\mathbf{u}_{j}$ represents the displacement at node $j$, but it need not be so for the derivation of CGMD. We will refer to $\mathbf{u}_{j}$ as nodal displacements, bearing in mind that the coarse-grained fields could be more general. Ultimately the usual criteria to ensure well-behaved numerics will apply, such as the cells should not have high aspect ratios and the mesh size should not change too abruptly; for the purposes of the formulation, the only requirement we impose is that if a region of the mesh is at the atomic scale, the positions of the nodes coincide with equilibrium lattice sites. This is not required for coarser regions of the mesh.

To the first approximation, CGMD is governed by mass and stiffness matrices. They are derived from the underlying atomistic physics, described by a molecular dynamics (MD) model (Allen and Tildesley, 1987). Define the discrete shape functions by evaluating the shape function $N_{j}(\mathbf{x})$ at the equilibrium lattice site $\mathbf{x}_{0 \mu}$ of atom $\mu$ :

$$
N_{j \mu}=N_{j}\left(\mathbf{x}_{0 \mu}\right)
$$

The discrete shape functions allow us to approximate the atomic displacements $\mathbf{u}_{\mu} \approx \sum_{j} \mathbf{u}_{j} N_{j \mu}$. If we were to make this a strict equality, we would be on the path to the Quasicontinuum technique. Instead, we consider this a constraint on the system, and allow all of the unconstrained degrees of freedom in the system to fluctuate in thermal equilibrium.

In particular, we demand that the interpolating fields be best fits to the underlying atomistic degrees of freedom of the system. In the case of the displacement field this requirement means 


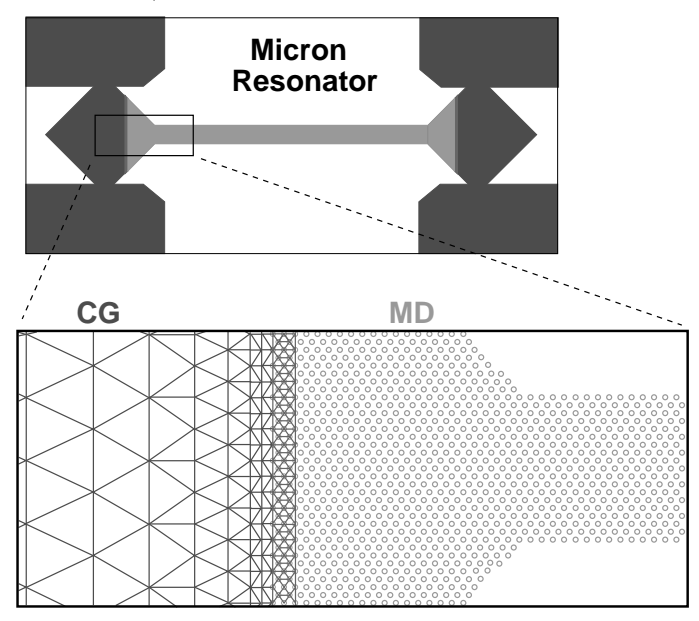

Figure 1: Schematic diagram of a concurrent multiscale simulation of a NEMS silicon microresonator $^{4-6}$ to illustrate how a system may be decomposed into atomistic (MD) and coarse-grained ( $\mathrm{CG}$ ) regions. The $\mathrm{CG}$ region comprises most of the volume, but the MD region contains most of the simulated degrees of freedom. Note that the CG mesh is refined to the atomic scale where it joins with the MD lattice.

that the nodal displacements minimize the chi-squared error of the fit:

$$
\chi^{2}=\sum_{\mu}\left|\mathbf{u}_{\mu}-\sum_{j} \mathbf{u}_{j} N_{j \mu}\right|^{2}
$$

The minimum of $\chi^{2}$ is given by

$$
\mathbf{u}_{j}=\left(N N^{T}\right)_{j k}^{-1} N_{k \mu} \mathbf{u}_{\mu} \equiv f_{j \mu} \mathbf{u}_{\mu}
$$

where repeated indices are summed and the inverse is a matrix inverse. We have introduced the weighting function expressed in terms of the discrete shape function as $f_{j \mu}=$ $\left(N N^{T}\right)_{j k}^{-1} N_{k \mu}$. Equation (4) provides the needed correspondence between the coarse and fine degrees of freedom.

Once the weighting function $f_{j \mu}$ is defined, the CGMD energy is defined as an average energy over the ensemble of systems in different points in phase space satisfying the correspondence relation (4). Mathematically, this is expressed as

$$
E\left(\mathbf{u}_{k}, \dot{\mathbf{u}}_{k}\right)=Z^{-1} \int d \mathbf{x}_{\mu} d \mathbf{p}_{\mu} H_{M D} e^{-\beta H_{M D}} \Delta,
$$

where $Z$ is the constrained partition function (the same integral without the $H_{M D}$ pre-

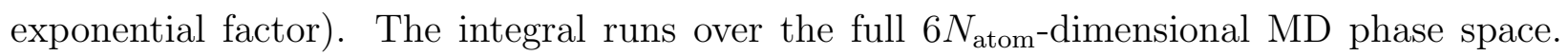
The inverse temperature is given by $\beta=1 / k T$. The factor $H_{M D}$ is the MD Hamiltonian, 
the sum of the atomistic kinetic and potential energies. The potential energy is determined by an interatomic potential, a generalization of the well-known Lennard-Jones potential that typically includes non-linear many-body interactions (Allen and Tildesley, 1987). The factor $\Delta$ is a product of delta functions enforcing the constraint,

$$
\Delta=\prod_{j} \delta\left(\mathbf{u}_{j}-\sum_{\mu} \mathbf{u}_{\mu} f_{j \mu}\right) \delta\left(\dot{\mathbf{u}}_{j}-\sum_{\mu} \frac{\mathbf{p}_{\mu} f_{j \mu}}{m_{\mu}}\right) .
$$

Once the energy (5) is determined, the equations of motion are derived as the corresponding Euler-Lagrange equations.

The CGMD energy (5) consists of kinetic and potential terms. The CGMD kinetic energy can be computed exactly using analytic techniques for any system; the CGMD potential energy can also be calculated exactly, provided the MD interatomic potential is harmonic. Anharmonic corrections may be computed in perturbation theory. The details are given in Rudd and Broughton (2004). Here we focus on the harmonic case, in which the potential energy is quadratic in the atomic displacements, and the coefficient of the quadratic term (times 2) is known as the dynamical matrix, $D_{\mu \nu}$. The result for harmonic CGMD is that

$$
\begin{aligned}
E\left(\mathbf{u}_{k}, \dot{\mathbf{u}}_{k}\right) & =U_{\text {int }}+\frac{1}{2}\left(M_{j k} \dot{\mathbf{u}}_{j} \cdot \dot{\mathbf{u}}_{k}+\mathbf{u}_{j} \cdot K_{j k} \mathbf{u}_{k}\right) \\
U_{\text {int }} & =N_{\text {atom }} E^{\text {coh }}+3\left(N_{\text {atom }}-N_{\text {node }}\right) k T \\
M_{i j} & =m N_{i \mu} N_{j \mu} \\
K_{i j} & =\left(f_{i \mu} D_{\mu \nu}^{-1} f_{j \nu}\right)^{-1} \\
& =N_{i \mu} D_{\mu \nu} N_{j \nu}-D_{i \mu}^{\times} \tilde{D}_{\mu \nu}^{-1} D_{j \nu}^{\times}
\end{aligned}
$$

where $M_{i j}$ is the mass matrix and $K_{i j}$ is the stiffness matrix. Here again and throughout this Article a sum is implied whenever indices are repeated on one side of an equation unless otherwise noted. The internal energy $U_{\text {int }}$ includes the total cohesive energy of the system, $N_{\text {atom }} E^{\text {coh }}$, as well as the internal energy of a collection of $\left(N_{\text {atom }}-N_{\text {node }}\right)$ harmonic oscillators at finite temperature. The form of the mass matrix (9) assumes a monatomic lattice. A more general form is given in Rudd and Broughton (2004). The two forms of the stiffness matrix are equivalent in principle, although in practice numerical considerations have favored one form or the other for particular applications. The first form (10) was used for the early CGMD applications. It is most suited for applications in which the nodal index may be Fourier transformed, such as the computation of phonon spectra. The second form (11) is better suited for real space applications. It depends on an off-diagonal block of the dynamical matrix

$$
D_{j \mu}^{\times}=\left(\delta_{\mu \rho}-N_{j \mu} f_{j \rho}\right) D_{\rho \nu} N_{j \nu}
$$

and a regularized form of the lattice Green function $\tilde{D}_{\mu \nu}^{-1}$ for the internal degrees of freedom 
that is defined in Rudd and Broughton (2004). Note that the mass matrix and the compliance matrix (the inverse of the stiffness matrix) are weighted averages of the corresponding MD quantities, the MD mass and MD lattice Green function, respectively.

The CGMD equations of motion are derived from the CGMD Hamiltonian (5) using the Euler-Lagrange procedure

$$
M_{j k} \ddot{\mathbf{u}}_{k}=-K_{j k} \mathbf{u}_{k}+\mathbf{F}_{j}^{\mathrm{ext}}
$$

where we have included the possibility of an external body force on node $j$ given by $\mathbf{F}_{j}^{\text {ext }}$. The anharmonic corrections to these equations of motion form an infinite Taylor series in powers of $\mathbf{u}_{k}$ (Rudd and Broughton, 2004). In regions of the mesh refined to the atomic level, it has been shown that the infinite series sums up to the MD interatomic forces; i.e., the original MD equations of motion are recovered in regions of the mesh refined to the atomic scale (Rudd and Broughton, 1998). In the case of a harmonic system, the recovery of the MD equations of motion in the atomic limit should be clear from the equations for the mass and stiffness matrices. In this limit $N_{i \mu}=\delta_{i \mu}$ and $f_{i \mu}=\delta_{i \mu}$, so $M_{i j}=m \delta_{i j}$ and $K_{i j}=D_{i j}$ from Eqs. (9) and (10), respectively. In practice, we define two complementary regions of the simulation. In the coarse-grained (CG) region, the harmonic CGMD equations of motion (13) are used, whereas in the region of the mesh refined to the atomic level, called the MD region, the anharmonic terms are restored through the use of the full MD equations of motion. In a CGMD simulation the mass and stiffness matrices are calculated once at the beginning of the simulation. The reciprocal space (Fourier transform) representation of the dynamical matrix is used in order to make the calculation of the stiffness matrix tractable. This representation implicitly assumes that the solid in the form of a crystal lattice free from defects in the CG region.

The CGMD mass matrix involves couplings between nearest neighbor nodes in the CG region, just as the distributed mass matrix of finite element modeling does. The fact that the mass matrix is not diagonal is inconvenient, since a system of equations must be solved in order to determine the nodal accelerations. The system of equations is sparse, but this step introduces some computational overhead, and it is desirable to eliminate it. In FEM, the distributed mass matrix is often replaced by a diagonal approximation, the lumped mass matrix (Hughes, 2000). In CGMD, the lumped mass approximation,

$$
M_{i j}^{\text {lump }}=m \delta_{i j} \sum_{\mu} N_{i \mu} \quad(\text { no sum on } i)
$$

has proven useful in the same way (Rudd and Broughton, 2000). This definition assumes that the shape functions form a partition of unity, so that $\sum_{i} N_{i \mu}=1$ for all $\mu$.

In principle, the determination of the equations of motion together with the relevant initial and boundary conditions completely specifies the problem. In practice, we have typically 
used a thermalized initial state and a mixture of periodic and free boundary conditions suitable for the problem of interest. The equations of motion are integrated in time using a velocity Verlet time integrator (Allen and Tildesley, 1987) with the conventional MD time step used throughout the simulation. The natural time scale of the CG nodes is longer due to the greater mass and greater compliance of the larger cells, and it would be natural to use a longer time step in the $\mathrm{CG}$ region. We have found little motivation to explore this possibility, however, since the computational cost of our simulations is typically dominated by the MD region, so there is little to gain by speeding up the computation in the CG region.

We turn now to the question of how CGMD simulations are analyzed. Much of the analysis of CGMD simulations is accomplished using standard MD techniques. The simulations are typically constructed such that the most interesting phenomena occur in the MD region, and here most of the usual MD tools may be brought to bear. Thermodynamic quantities are calculated in the usual way, and the identification and tracking of crystal lattice defects may be accomplished with conventional techniques.

In some cases it may be of interest to analyze the simulation in the CG region, as well. For example, it may be of interest to plot the temperature throughout the simulation in order to verify that the behavior at the $\mathrm{MD} / \mathrm{CG}$ interface is reasonable. In MD the temperature is directly related to the mean kinetic energy of the atoms: $k T=\frac{1}{3} m\left\langle|\dot{\mathbf{u}}|^{2}\right\rangle$, where the brackets indicate the average (Allen and Tildesley, 1987). In CGMD, a similar expression holds (Rudd and Broughton, 2004)

$$
k T=\frac{1}{3}\left\langle\left|\dot{\mathbf{u}}_{i}\right|^{2}\right\rangle / M_{i i}^{-1} \quad(\text { no sum on } i)
$$

where $M_{i i}^{-1}$ is the diagonal component corresponding to node $i$ of the inverse of the mass matrix. This analysis of the temperature and thermal oscillations is closely tied to the kinetic energy in the CG region. Similar tools are available to analyze the potential energy and the related quantites such as deformation, pressure and stress (Rudd and Broughton, 2004).

\section{Validation}

Validation of concurrent multiscale models is a challenge in its own right, and the development of quantitative tools and performance measures to analyze models like CGMD has taken place at the same time as the development of the first models. CGMD has been tested in several ways to see how it compares with a full MD simulation of a test system, as well as other concurrent multiscale simulations. The first test was the calculation of the spectrum of elastic waves or phonons. The techniques to calculate these spectra in atomistic systems have been developed long ago in the field of lattice dynamics (Born and Huang, 1954). In general the phonon spectrum is comprised of $D$ acoustic mode branches (where $D$ is the number of dimensions) together with $D\left(N_{\text {unit }}-1\right)$ optical branches (where $N_{\text {unit }}$ is the num- 
ber of atoms in the elementary unit cell of the crystal lattice) (Ashcroft and Mermin, 1976). The acoustic modes are distinguished by the fact that their frequency goes to zero as their wavelength becomes large. The infinite wavelength corresponds to uniform translation of the system, a process that costs no energy and hence corresponds to zero frequency.

Elastic wave spectra are an interesting test of CGMD and other concurrent multiscale techniques because they represent a test of dynamics and because elastic waves have a natural length scale associated with them: the wavelength. When a coarse-grained mesh is introduced, the shortest wavelengths are excluded. These modes are eliminated because they are irrelevant in the CG region, and their elimination increases the efficiency of the simulation. The test then is to see how well the model describes those longer wavelength modes that are represented in the $\mathrm{CG}$ region.

The elastic wave spectra for solid argon were computed in CGMD on a uniform mesh for various mesh sizes, and compared to the MD spectra and spectra computed using a FEM model based on continuum elasticity (Rudd and Broughton, 2000, 2004). The bonds between argon atoms were modeled with a Lennard-Jones potential cut off at the fifth shell of neighboring atoms. Several interesting results were found. First, both CGMD and FEM agreed with the MD spectrum at long wavelengths. This is to be expected, since for wavelengths much longer than the mesh spacing, the waveform should be well represented on the mesh. Also, at long wavelengths the FEM assumption of a continuous medium is justified, and the slope of the spectrum gives the sound velocity, $c=\omega / k$ for $k \rightarrow 0$. Here $\omega$ is the (angular) frequency and $k$ is the wave number. The error in $\omega(k)$ was found to be of order $\mathcal{O}\left(k^{2}\right)$ for FEM, as expected. It goes to zero in the long wavelength limit, $k \rightarrow 0$. One nice feature of CGMD was a reduced error of order $\mathcal{O}\left(k^{4}\right)$ (Rudd and Broughton, 1998). Moreover, CGMD provides a better approximation of the elastic wave spectra for all wavelengths supported on the mesh. Of course, CGMD also has the important feature that the elastic wave spectra are reproduced exactly when the mesh is refined to the atomic level, a property that FEM does not possess. Interatomic forces are not merely FEM elasticity on an atomic sized grid.

Solid argon forms a face-centered cubic crystal lattice and hence has only three acoustic wave branches in its phonon spectrum. For crystals with optical phonon branches, there is more than one way to implement the coarse-graining, depending on the physics that is of interest, but the general CGMD framework continues to work well (Kraczek, 2003).

The other validation of CGMD has been the study of the reflection of elastic waves from the MD/CG interface. For applications such as crack propagation, it has proven important to control this unphysical reflection. The reflected waves can propagate back into the heart of the MD simulation and interfere with the processes of interest. In the case of crack propagation, a noticeable anomaly in the crack speed occurs at the point in time when the reflected waves reach the crack tip (Holian and Ravelo, 1995).

The reflection coefficient, a measure of the amount of elastic wave energy reflected at a given 

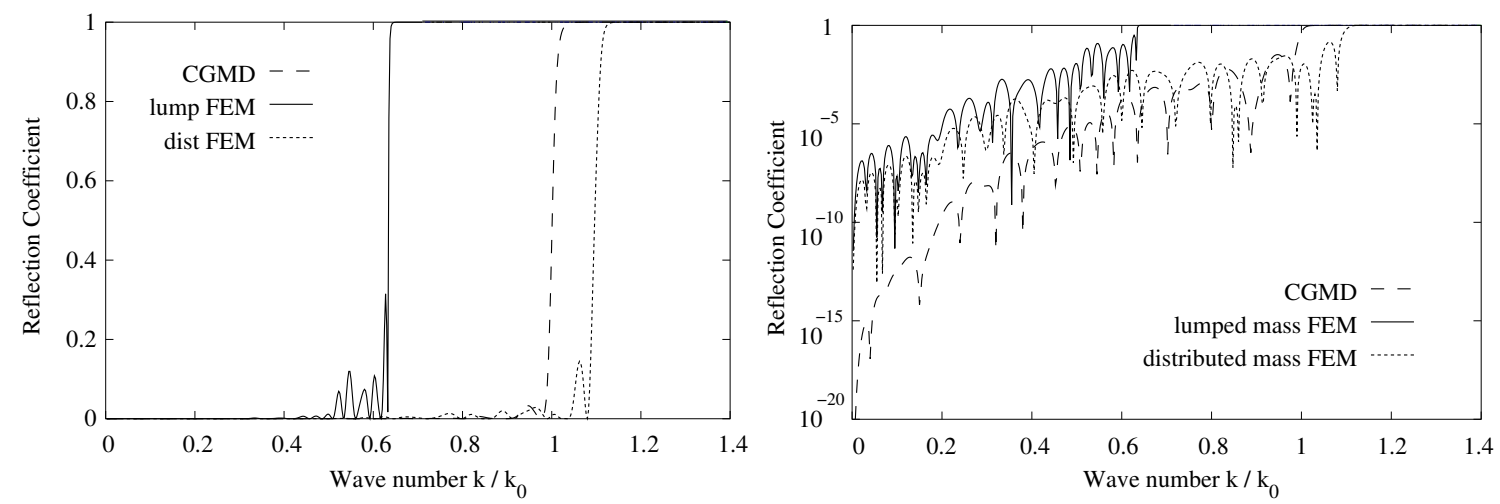

Figure 2: A comparison of the reflection of elastic waves from a CG region in three cases: CGMD and two varieties of FEM. Note that the reflection coefficient is plotted on a log scale. A similar graph plotted on a linear scale is shown in Rudd and Broughton (1998). The dashed line marks the natural cutoff $\left[k_{0}=\pi /\left(N_{\max } a\right)\right]$, where $N_{\max }$ is the number of atoms in the largest cells. The bumps in the curves are scattering resonances. Note that at long wavelengths CGMD offers significantly suppressed scattering.

wavelength, has been calculated for CGMD and FEM based on continuum elasticity (Rudd and Broughton, 1998, 2004). Typical results are shown in Figure 2. Long wavelength elastic waves are transmitted into the CG region, whereas short wavelength modes are reflected. The short wavelengths cannot be supported on the mesh, and since energy is conserved, they must go somewhere and they are reflected. The transmission threshold is expected to occur at a wave number $k_{0}=\pi /\left(N_{\max } a\right)$. The CGMD threshold occurs precisely at this wave number, while the threshold for transmission in distributed mass and lumped mass FEM models occurs somewhat above and below this value, respectively.

The scattering in the long wavelength limit shows a generalized Rayleigh scattering behavior. In conventional Rayleigh scattering the scattering cross-section goes like $\sigma \sim k^{4}$, which is the behavior exhibited by scattering here in FEM. For CGMD, the scattering drops off more quickly at long wavelengths, with the reflection coefficient approximately proportional to $k^{8}$ (Rudd and Broughton, 2004).

One aspect of concurrent multiscale modeling that remains poorly understood is the requirements for a suitable mesh. Certainly, many of the desired properties are clear either from the nature of the problem or from experience with FEM. For example, the mesh needs to be refined to the atomic level in the MD region, so here the mesh nodes should coincide with equilibrium crystal lattice sites. In the periphery large cells are desirable since the gain in efficiency is proportional to the cell size. From FEM it is well known that the aspect ratio of the cells should not be too large. Beyond these basic criteria, one is left with the task of generating a mesh that interpolates between the atomic-sized cells in the MD region to 

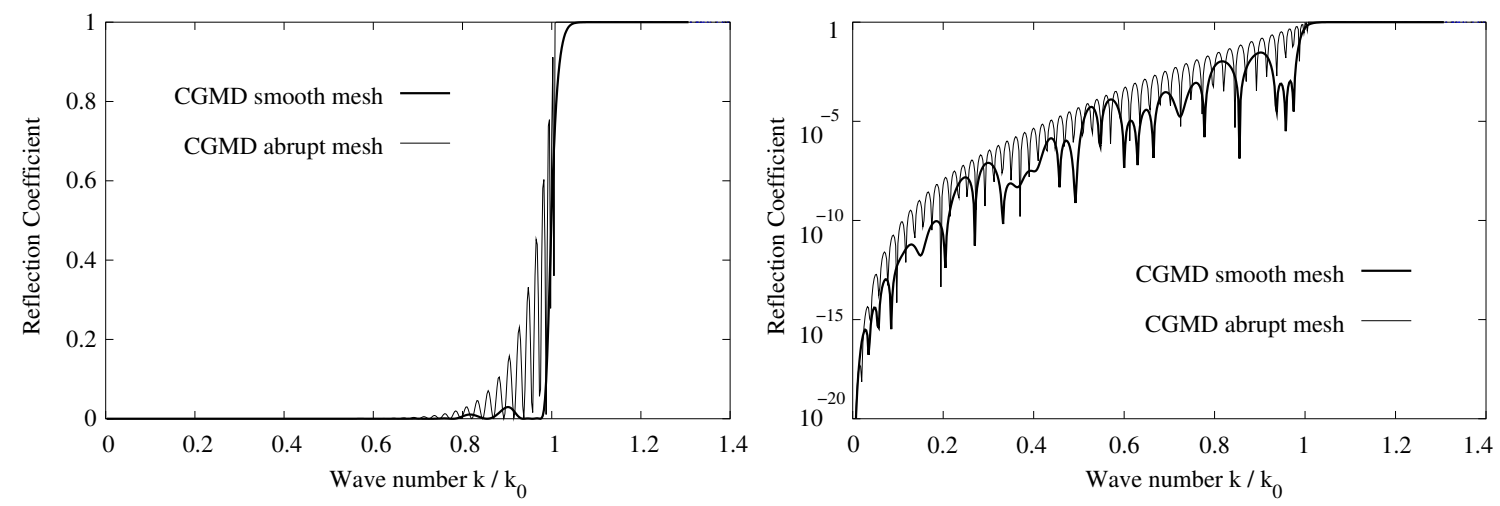

Figure 3: A comparison of the reflection of elastic waves from a coarse-grained region whose mesh varies smoothly in cell size and one with an abrupt change in cell size, both computed in CGMD. In both cases the reflection coefficient is plotted as a function of the wave number in units of the natural cutoff $\left[k_{0}=\pi /\left(N_{\max } a\right)\right]$, where $a$ is the lattice constant and $N_{\text {max }} a=20 a$ is the maximum linear cell size in the mesh. The pronounced series of scattering resonances in the case of the abruptly changing mesh is undesirable. The second panel is a log-linear plot of the same data in order to show how the series of scattering resonances continues at decreasing amplitudes to long wavelengths.

the large cells in the periphery without introducing high aspect ratio cells. One question we have investigated is whether the abruptness of this transition matters, and indeed it does matter.

Figure 3 shows the reflection coefficient as a function of the wave number for two meshes that go between an MD region and a CG region with a maximum cell size of 20 lattice spacings. In one case, the transition is made gradually, whereas in the other case it is made abruptly. The mesh with the abrupt transition exhibits markedly increased scattering, including a series of strong scattering resonances. Note that the envelope of the scattering curve is well defined in the case of the abrupt mesh, a property used to calculate the scaling of the reflection coefficient, $\mathcal{R} \sim k^{8}$.

\section{Outlook}

CGMD provides a formalism for concurrent multiscale modeling at finite temperature. The initial tests have been very encouraging, but there are still many ways in which CGMD can be developed. One area of active research is numerical algorithms to make CGMD more efficient for large simulations. The calculation of the stiffness matrix involves the inverse of a large matrix whose size grows with the number of nodes in the CG region, $N_{\mathrm{CGnode}}$. The calculation of the inverse scales like $N_{\text {CGnode }}^{3}$ and the matrix storage scales like $N_{\text {CGnode }}^{2}$, for the exact matrix without any cutoff. Even though the calculation of the stiffness matrix need 
only be done once during the simulation, the calculation has proven sufficiently onerous to prevent the application of CGMD to the large-scale simulations for which it was originally intended. Only now are linear scaling CGMD algorithms starting to become available.

There are several directions in which CGMD has begun to be extended for specific applications. The implementation of CGMD described in this Article conserves energy. It implicitly makes the assumption that the only thermal fluctuations that are relevant to the problem are those supported on the mesh. Fluctuations of the degrees of freedom that have been integrated out are neglected. Those fluctuations can be physically relevant in several ways (Rudd, 2002). First, they exert random and dissipative forces on the coarse-grained degrees of freedom in a process that is analogous to the forces in Brownian motion exerted on a large particle by the atoms in the surrounding liquid. Second, they also act as a heat bath that is able to exchange and transport thermal energy. Finally, they can transport energy in non-equilibrium processes, such as the waves generated by a propagating crack discussed above.

A careful treatment of the coarse-grained system leads to a generalization of the CGMD equations of motion presented above (Rudd, 2002). In addition to the conservative forces, there are random and dissipative forces that form a generalized Langevin equation. The dissipative forces involve a memory function in time and space that acts to absorb waves that cannot be supported in the CG region. The memory kernel is similar to those that have been discussed in the context of absorbing boundary conditions for MD simulations (Cai et al., 2000; E and Huang, 2001), except that in CGMD the range of the kernel is shorter because the long wavelength modes are able to propagate into the CG region and do not need to be absorbed. Interestingly, in the case of a CG region surrounded by MD regions, the memory kernel also contains propagators that recreate the absorbed waves on the far side of the CG region after the appropriate propagation delay (Rudd, 2002). Of course, use of the generalized Langevin incurs additional computational expenses both in terms of run time and memory.

There are many other ways in which CGMD could be extended. Additional coarse-grained fields could be introduced to model various material phenomena such as electrical polarization, defect concentrations and local temperature. Fluxes such as heat flow and defect diffusion can be included through the technique of coarse-graining the atomistic conservation equations. CGMD provides a powerful framework in which to formulate finite temperature multiscale models for a variety of applications.

\section{Acknowledgments}

This article was prepared under the auspices of the U.S. Department of Energy by University of California, Lawrence Livermore National Laboratory under Contract W-7405-Eng-48. 


\section{Bibliography}

Abraham, F. F., Broughton, J. Q., Kaxiras, E. and Bernstein, N., 1998. Spanning the length scales in dynamic simulation. Comput. in Phys. 12, 538-546.

Abraham, F. F., Walkup, R., Gao, H., Duchaineau, M., Diaz de la Rubia, T. and Seager, M., 2002. Simulating materials failure by using up to one billion atoms and the world's fastest computer: Work-hardening. Proc. Natl. Acad. Sci. USA 99, 5783-5787.

Allen, M. P. and Tildesley, D. J., 1987. Computer Simulation of Liquids. Clarendon Press, Oxford.

Ashcroft, N. W. and Mermin, N. D., 1976. Solid State Physics. Saunders College Press, Philadelphia.

Averback, R. S. and Diaz de la Rubia, T., 1998. Fundamental Studies of Radiation Effects in Solids. Solid State Physics 51, 281-402.

Born, M. and Huang, K., 1954. Dynamical Theory of Crystal Lattices. Clarendon Press, Oxford.

Broughton, J. Q., Abraham, F. F., Bernstein, N. and Kaxiras, E., 1999. Concurrent coupling of length scales: Methodology and application. Phys. Rev. B 60, 2391-2403.

Cai, W., de Koning, M., Bulatov, V. V. and Yip, S., 2000. Minimizing Boundary Reflections in Coupled-Domain Simulations. Phys. Rev. Lett. 85, 3213-3216.

Curtarolo, S. and Ceder, G., 2002. Dynamics of an Inhomogeneously Coarse Grained Multiscale System. Phys. Rev. Lett. 88, 255504.

Curtin, W. A. and Miller, R. E., 2003. Atomistic/Continuum Coupling in Computational Materials Science. Modelling Simul. Mater. Sci. Eng. 11, R33-R68.

E, W. and Huang, Z., 2001. Matching Conditions in Atomistic-Continuum Modeling of Materials. Phys. Rev. Lett. 87, 135501.

Holian, B. L. and Ravelo, R., 1995. Fracture simulations using large-scale moleculardynamics. Phys. Rev. B 51, 11275-11288.

Hughes, T. J. R., 2000. The Finite Element Method: Linear Static and Dynamic Finite Element Analysis. Dover, Mineola.

Kohlhoff, S., Gumbsch, P. and Fischmeister, H. F., 1991. Crack-propagation in BCC crystals studied with a combined finite-element and atomistic model. Philos. Mag. A 64, 851-878.

Kraczek, B., 2003. private communication . 
Mason, D. R., Rudd, R. E. and Sutton, A. P., 2004. Atomistic modelling of diffusional phase transformations with elastic strain. submitted to J. Phys.: Condens. Matter.

Moriarty, J. A., Belak, J. F., Rudd, R. E., Soderlind, P., Streitz, F. H. and Yang, L. H., 2002. Quantum-based atomistic simulation of materials properties in transition metals. $J$. Phys.: Condens. Matter 14, 2825-57.

Rudd, R. E., 2002. Coarse-Grained Molecular Dynamics: Dissipation due to Internal Modes. Mat. Res. Soc. Symp. Proc. 695, T10.2.

Rudd, R. E., Briggs, G. A. D., Sutton, A. P., Medieros-Ribiero, G. and Williams, R. S., 2003. Equilibrium model of bimodal distributions of epitaxial island growth. Phys. Rev. Lett. 90, 146101.

Rudd, R. E. and Broughton, J. Q., 1998. Coarse-grained molecular dynamics and the atomic limit of finite elements. Phys. Rev. B 58, R5893-R5896.

Rudd, R. E. and Broughton, J. Q., 1999. Atomistic Simulation of MEMS Resonators through the Coupling of Length Scales. J. Modeling and Simul. Microsys. 1, 29-38.

Rudd, R. E. and Broughton, J. Q., 2000. Concurrent Multiscale Simulation of Solid State Systems. Phys. Stat. Sol. (b) 217, 251-291.

Rudd, R. E. and Broughton, J. Q., 2004. Coarse-grained molecular dynamics:Non-linear finite elements and finite temperature. in preparation for Phys. Rev. B .

Shilkrot, L. E., Miller, R. E. and Curtin, W. A., 2002. Coupled atomistic and discrete dislocation plasticity. Phys. Rev. Lett. 89, 025501.

Tadmor, E. B., Ortiz, M. and Phillips, R., 1996. Quasicontinuum analysis of defects in solids. Philos. Mag. A 73, 1529-1563.

Townsend, A. A., 1976. The Structure of Turbulent Shear Flow. 2nd edn. Cambridge Univ. Press, Cambridge.

Robert E. Rudd

Lawrence Livermore National Laboratory

University of California, L-045

Livermore, CA 94551 USA

robert.rudd@llnl.gov 


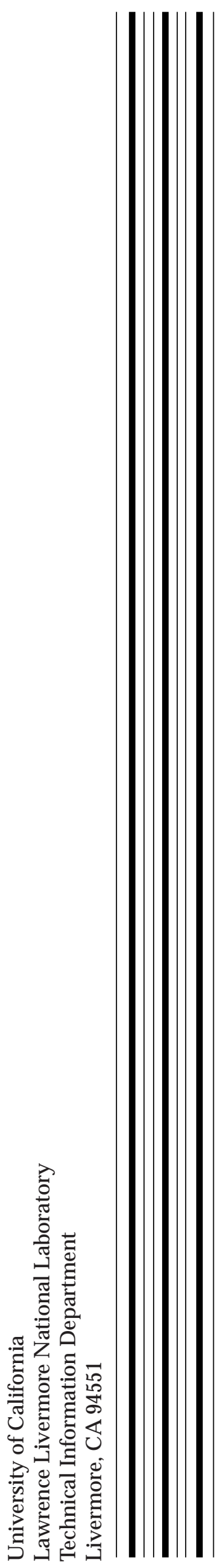

